\title{
Ontology of a Wavefunction from the Perspective of an Invariant Proper Time
}

\author{
Salim Yasmineh * \\ University of Paris, Paris, France
}

All the arguments of a wavefunction are defined at the same instant, implying the notion of simultaneity. In a somewhat related matter, certain phenomena in quantum mechanics seem to have non-local causal relations. Both concepts contradict the special relativity. We propose defining the wavefunction with respect to the invariant proper time of special relativity instead of the standard time. Moreover, we shall adopt the original idea of Schrodinger, suggesting that the wavefunction represents an ontological cloud-like object that we shall call "individual fabric" that has a finite density amplitude vanishing at infinity. Consequently, the action of measurement can be assimilated to the introduction of a confining potential that triggers an inherent nonlocal mechanism within the individual fabric. This mechanism is formalised by multiplying the wavefunction with a localising Gaussian, as in the GRW theory, but in a deterministic manner.

\section{OPEN ACCESS}

Edited by:

Jinjin $L i$,

Shanghai Jiao Tong University, China

Reviewed by:

William Sulis,

McMaster University, Canada

Luca Nanni,

University of Ferrara, Italy

${ }^{*}$ Correspondence:

Salim Yasmineh

sayasmineh@gmail.com

Specialty section:

This article was submitted to

Mathematical and Statistical Physics,

a section of the journal

Frontiers in Physics

Received: 04 September 2021

Accepted: 19 November 2021

Published: 22 December 2021

Citation:

Yasmineh S (2021) Ontology of a Wavefunction from the Perspective of

an Invariant Proper Time.

Front. Phys. 9:770764.

doi: 10.3389/fphy.2021.770764
Keywords: proper time, non-locality, simultaneity, wavefunction, measurement

\section{INTRODUCTION}

As clearly explained by Travis Norsen in his book "Foundations of Quantum Mechanics" [1] there are three main interconnected problems in Quantum Mechanics: measurement, relativity (nonlocality and simultaneity) and ontology of a wavefunction.

Quantum measurements can be highlighted by measuring the physical properties of a microscopic system using a measuring instrument. Suppose the quantum mechanical wavefunction describing the microscopic system is in a superposition of the eigenstates of an operator corresponding to the physical property that is being measured. Then, contrary to real observations, the Schrodinger equation evolution implies that the measuring instrument should be in a macroscopic superposition of many distinct states. Standard quantum mechanics (Copenhagen interpretation) solves this problem of macroscopic superposition by introducing Born's rule. This rule postulates that a wavefunction evolves deterministically in accordance with Schrodinger's equation, except during measurement when it collapses with a certain probability measure to one particular eigenstate. The measurement problem consists of understanding this paradoxical transition from a deterministically evolving spread-out wavefunction into a sudden probabilistic localisation.

The relativity problem concerns two main interrelated features. The first relates to the relativity of simultaneity which is a consequence of Lorentz invariance, and the second relates to the notion of relativistic locality. In quantum mechanics, all the arguments of a wavefunction are defined at the same instant, thus requiring the notion of absolute simultaneity. For example, a quantum system composed of a pair of entangled particles behaves in such a manner that the quantum state of one particle cannot be described independently of the state of the other. Standard quantum mechanics 
postulates that neither one of the particles has a determinate state until it is measured. As both particles are correlated, it is necessary that when the state of one particle is measured, the second particle should "simultaneously" acquire a determinate state. However, the laws of physics are invariant under Lorentz transformations. There is no meaning of "simultaneity" independently of any frame of reference, and there should be no preferred frame of Refs. $[2,3]$. On the other hand, the nonlocality problem can be resumed by Einstein's argument that if quantum mechanics is complete, then the collapse of the wave function is a dynamical process that conflicts with relativistic locality. This quantum phenomenon was first introduced as a thought experiment in the EPR paper [4], and it was later discovered that it can be experimentally testable by using Bell's inequality $[5,6]$. Numerous experiments, such as Aspect's experiment [7], proved the validity of quantum entanglement and hence a certain notion of non-local connections. The notions of nonlocality and simultaneity are thus inferred by quantum mechanics while being forbidden by the postulates of relativity. The notions of non-locality, causation, and time in QM are discussed in the papers of L. Felline and K. Thomsen [8, 9].

Finally, the ontological problem concerns the fact that for more than one particle, the wavefunction is defined on a highdimensional configuration space and not on a physical space, and thus, cannot represent a physically real field. For a physical ontology, the wavefunction should be the representation of a real physical entity.

In addition to the Copenhagen interpretation, there are several candidate theories that propose solutions to at least some of the above problems. Drummond [10] provided a conceptual analysis of these different theories with deep critical insight. The main current theories comprise the Everett many-world theory [11], de Broglie Bohm pilot-wave theory [12, 13], and the GRW spontaneous collapse theory [14]. This study is primarily related to the last two theories.

Everett's many-world theory [11] discards the collapse postulate, and all measurement results exist but in different worlds. In line with this interpretation, it has been claimed [15] that when a measurement is conducted on a particle in a superposition state, a deterministic branching takes place where, on one branch, a first detector detects the particle while a second detector does not, and at the same time, but on the other branch (i.e. another world), the first detector does not detect the particle while the second detector detects it. However, this interpretation pauses probabilistic and ontological problems. In particular, the axioms of quantum mechanics say nothing about the existence of multiple physical worlds [16]. Greaves [17] provided a detailed account of the probabilistic aspects of this theory.

The de Broglie Bohm theory is very promising and is discussed in detail by Jean Bricmont in his book "Making Sense of Quantum Mechanics" [18] and in Norsen [1] as well as in the Symposium Louis de Broglie, and in particular, in the introduction to this symposium [19]. The basic idea behind this theory is that a corpuscle, such as an electron, always has a well-determined position on a definite trajectory through physical space. However, its movement is influenced by an associated wavefunction, giving rise to wave-like properties. Thus, according to this theory, an electron is a particle "and" a wave. The de Broglie Bohm theory solves the measurement problem and accounts for nonlocality. However, this does not seem to solve the problem of simultaneity. For a multi-particle system, the theory explicitly formulates the dependence of a particle's evolution at a given instant on the positions of all the other particles at the same instant. Moreover, the ontology of the wavefunction which is defined in the configuration space, remains unclear. However, de Broglie tried to develop [20] a version of the theory according to which interactions between quantum systems are not in the configuration space but in the real three-dimensional physical space.

The GRW spontaneous collapse theory [13] was discussed by Norsen [1]. It modifies Schrodinger's equation with stochastic terms that has the effect of making a wavefunction obeys Schrodinger's equation most of the time, except for exceedingly rare and random instants when it undergoes a spontaneous collapse. GRW solves the measurement problem, accounts for non-locality, and provides a physical explanation of the wavefunction. Nevertheless, it does not solve the problem of simultaneity and seems to be ad hoc.

Mielnik [21] showed that an apparent paradox is produced when attempting to reconcile the instantaneous collapse of the wavefunction with the requirements of special relativity. What is instantaneous in one Lorentz frame is not necessarily instantaneous in the other.

Helwig and Kraus [22] provided a formal description of the field measurements according to a covariant description of the relativistic measurement process. They consider a formalism in which the field state remains unchanged in the backward light cone of a finite spacetime region and changes in the forward and side light cones. They advocated the idea that collapse should occur along the backward light cone of the measurement. This picture has been criticised by Aharonov and Albert [23, 24].

Finkelstein [25] illustrates the difficulty of reconciling the collapse with special relativity with the following two assumptions:

A1: A quantum system is represented by a state vector which does not depend on the Lorentz from which the system is described.

A2: When the quantum system is measured, the state vector does, in general, collapse; that is, the state vector will not be independent of time.

Finkelstein [25] considers that both assumptions A1 and A2 can be maintained at the cost of allowing the state vector to depend on the position from which it is described. Finkelstein [25] advocated the idea that it is not meaningful to talk about the state (or probability density) at a given time in the absence of the position from which it is described. He outlines a procedure in which collapse occurs along the forward light cone of measurement, such that all observers at a given spacetime point will obtain the same density operator or state vector. Aharonov and Albert [23, 24] seem to consider that the state vector should not depend on the place from which it is described, and that assumption A1 should be abandoned. Mielnik [21], on 
the other hand, considers collapse as instantaneous and prefers to abandon A2.

In this paper, we point out that assumptions A1 and A2 are compatible for all observers when the state vector is defined with respect to the proper time instead of ordinary time.

\section{INVARIANT SPACETIME STRUCTURE}

We propose to consider the evolution of a particle from the perspective of proper time $\tau$, by using the hyperbolic spacetime structure inside a light cone associated with the particle. Specifically, we shall use the formalism of Minkowski spacetime [26] as defined in a geometrical manner by Eric Gourgoulhon in his book "Special Relativity in General frames" [27].

The Minkowski spacetime $\mathcal{M}$ is defined as an affine space of dimension 4 on $\mathrm{R}$ endowed with a bilinear metric tensor $\mathrm{g}$ defined in an underlying vector space $\mathrm{E}$ of signature $(+,-,-,-)$. In the vector space $\mathrm{E}$, a set $\mathrm{C}$ composed of the zero vector, and all null vectors form a light cone $\mathrm{C}$ composed of two sheets $\mathrm{C}^{+}$and $\mathrm{C}^{-}$ defining the future and past light cones, respectively.

Given the above defined spacetime $\mathcal{M}$ and given an arbitrary point $O \in \mathcal{M}$, a family of affine subspaces $\left(S_{\tau}\right)_{\tau \in R}$ is defined such that each subspace $S_{\tau}$ corresponds to the set of points of $\mathcal{M}$ that can be connected to $\mathrm{O}$ by a time-like vector $\overrightarrow{O N}$ of modulus $\tau$, where $\tau \in R$ :

$$
S_{\tau}=\left\{N \in \mathcal{M}, \overrightarrow{O N} \cdot \overrightarrow{O N}=-\tau^{2}<0\right\}
$$

We are henceforth interested in physical systems that follow time-like or null worldlines and do not consider the set of space-like vectors. In the spacetime $(\mathcal{M}, \mathrm{g})$, a point $N \in \mathcal{M}$ is said to belong to the subspace $S_{\tau}$ iff $\overrightarrow{O N} \cdot \overrightarrow{O N}=-\tau^{2}$. Each set of points $S_{\tau}$ consists of two subsets or two sheets, $S_{\tau}^{+}$and $S_{\tau}^{-}$ belonging to the interiors of the future $\mathrm{C}^{+}$and past $\mathrm{C}^{-}$light cones, respectively:

$$
\begin{array}{ll}
S_{\tau}^{+}=\left\{N \in S_{\tau},\right. & \tau \geq 0\} \\
S_{\tau}^{-}=\left\{N \in S_{\tau},\right. & \tau<0\}
\end{array}
$$

Let $\left(x^{0}, x^{1}, x^{2}, x^{3}\right)$ be the coordinates of $N \in S_{\tau}$ in the affine frame defined by origin $\mathrm{O}$ and basis $\left(e_{\alpha}\right)$, then $\overrightarrow{O N} \cdot \overrightarrow{O N}=-\tau^{2}$ can be expressed as follows:

$$
-\left(x^{0}\right)^{2}+\left(x^{1}\right)^{2}+\left(x^{2}\right)^{2}+\left(x^{3}\right)^{2}=-\tau^{2}
$$

Where $x^{o}=t, x^{1}=\frac{x}{c}, x^{2}=\frac{y}{c}, x^{3}=\frac{z}{c}$.

Equation 2.4 is a three-dimensional hyperboloid of the two sheets $S_{\tau}^{+}$and $S_{\tau}^{-}$spanned by the free extremities of the time-like vectors $\overrightarrow{O N}$.

The modulus $\tau$ of the time-like vector $\overrightarrow{O N}$ is the proper time for the physical system. It generates a family of affine subspaces $\left(S_{\tau}\right)_{\tau \in R}$ defined by (2.1). This family consists of threedimensional hyperboloids associated on one hand with futuredirected proper times $\tau \geq 0$ and on the other hand, with pastdirected proper times $\tau<0$.
The sheet $S_{\tau}$ of each hyperboloid forms a "spatialhypersurface" that we shall simply call "slice" associated with a corresponding proper time $\tau \in R$. All points on any given slice $S_{\tau}$ are associated with the same proper time $\tau$ which is indeed invariant to all observers from the perspective of any inertial frame of reference.

Without any loss of generality, we refer hereafter to the twodimensional space time. Let $(O ; x, t)$ be an orthonormal frame of reference defined by origin $\mathrm{O}$, a spatial $\mathrm{x}$-axis, and a temporal $\mathrm{t}$-axis. We take $\mathrm{c}=1$, then the light cone is composed of the lines $\mathrm{X}$ and $\mathrm{Y}$ (where $\mathrm{X}$ is defined by $t=x$ and $\mathrm{Y}$ is defined by $t=-x$ ) inside which are piled with a family of simple hyperbolas $\left(S_{\tau}\right)_{\tau \in R}$. Let $(x, t)$ be the coordinates of a point $\mathrm{M}$ on a given hyperbola $\left(M \in S_{\tau}\right)$. Then, similar to Eq. 2.4, $\overrightarrow{O M} \cdot \overrightarrow{O M}=-\tau^{2}$ can be expressed as follows:

$$
t^{2}-x^{2}=\tau^{2}
$$

Proper time $\tau$ is thus, given by the following expression:

$$
\tau= \pm \sqrt{t^{2}-x^{2}}
$$

Where the "+" sign corresponds to a future-directed proper time and the "-" sign corresponds to a past-directed proper time.

For each proper time $\tau$, the free extremity $\mathrm{M}$ of the vector $\overrightarrow{O M}$ (of modulus $\tau$ ) spans the hyperbolic-slice $S_{\tau}$. The hyperbolic slice $S_{\tau}$ is a piecewise twice continuously differentiable curve of the Minkowski spacetime $\left(\mathcal{M}_{I}, \mathrm{~g}\right)$ composed of a set of hyperbolic points $(u, \tau)$. The hyperbolic coordinate $u$ represents the orientation of a straight line (or a ray $R_{u}$ ) passing through the origin. All points $(u, \tau)$ on the same ray $R_{u}$ share the same hyperbolic coordinate $u$. On the other hand, all points $(u, \tau)$ on the same slice $S_{\tau}$ share the same invariant proper time coordinate $\tau$.

Thus, the rays $\left(R_{u}\right)_{u}$ and hyperbolic slices $\left(S_{\tau}\right)_{\tau}$ define a hyperbolic frame of reference $\left(O ; R_{u}, S_{\tau}\right)$ where a given hyperbolic point $(u, \tau)$ is the intersection between the corresponding ray $R_{u}$ and slice $S_{\tau}$.

The hyperbolic slice $S_{\tau}$ can be parameterised by a bijective function $\varphi$ from the points on the real axis $R$ into the points on that slice $S_{\tau}$ (i.e. : $R \rightarrow S_{\tau}$ ) such that any point $u$ on slice $S_{\tau}$ is given by $u=\varphi(\lambda) \equiv u(\lambda)$. The parameter $\lambda$ can be chosen as the standard time coordinate $t$ or standard space coordinate $x$.

The mapping between a point $(x, t)$ in the orthonormal frame of reference $(O ; x, t)$ and a hyperbolic point $(u, \tau)$ within the upper light cone in the hyperbolic frame of reference $\left(O ; R_{u}, S_{\tau}\right)$ is

$$
u=\ln \sqrt{\frac{t+x}{t-x}} \text { and } \tau=\sqrt{t^{2}-x^{2}}
$$

The inverse mapping of (2.7) can be expressed as follows:

$$
x=\tau \sinh u \text { and } t=\tau \cosh u
$$

As each slice $S_{\tau}$ is associated with a corresponding proper time $\tau$, then, all points $u$ belonging to that slice $S_{\tau}$ are said to be "simultaneous" in the sense of proper time. In other words, each slice $S_{\tau}$ is a class of "proper-time-simultaneity" made up of a set of points $u_{\tau}$ that are associated with the same proper time instant $\tau$. 
The passage from one slice $S_{\tau_{1}}$ into a subsequent slice $S_{\tau_{2}}$ represents the "transition" from the first proper time $\tau_{1}$ to a consequent proper time $\tau_{2}$. Proper time provides invariant time ordering of the set of slices.

We note that the passage from one slice $S_{\tau_{1}}$ into a subsequent slice $S_{\tau_{2}}$ expands the standard spatial separation between any two points. Indeed, let $\left(u_{a}, \tau_{1}\right)$ and $\left(u_{b}, \tau_{1}\right)$ be two different points at an initial proper time $\tau_{1}$, and $\left(u_{a}, \tau_{2}\right)$ and $\left(u_{b}, \tau_{2}\right)$ be the corresponding points at a subsequent proper time $\tau_{2}$, where $u_{a}$ and $u_{b}$ represent two different rays. As $\tau_{2}>\tau_{1}$, then, the standard spatial separation $\tau_{2} \sinh \left(u_{b}-u_{a}\right)$ at the later proper time $\tau_{2}$ is greater than the standard spatial separation $\tau_{1} \sinh \left(u_{b}-u_{a}\right)$ at the previous proper times $\tau_{1}$.

\section{WAVEFUNCTION AND ITS EVOLUTION THROUGH PROPER TIME}

In view of the above, we propose to define the wavefunction with respect to the proper time which is indeed the only real physical time. In general, the notion of proper time is defined with respect to the worldline, followed by a particle. However, in this study, we take advantage of the geometrical representation of the proper time, as described in $[3,27]$. Indeed, as all the points on a given hyperboloid or slice $S_{\tau}$ are equidistant from the origin of a Lorentz coordinate system, all these points have the same proper time.

We propose to define a unit state vector $|\varphi(\tau)\rangle$ as a function of proper time $\tau \in R$ by relating it to a slice $S_{\tau}$ which is globally associated with the corresponding proper time $\tau$. Thus, once the proper time $\tau$ is specified, the points of the slice $S_{\tau}$ may be defined by the set of points $u_{\tau}=(\tau, u)$ where $u$ is a hyperbolic variable or by the set of points $x_{\tau}=(\tau, x)$ where $x$ is a Cartesian variable. For a given proper time $\tau$, the variables $x$ and $u$ are one-to-one related according to Eq. 2.8.

For simplicity, we express a slice $S_{\tau}$ by the set of points $\left\{x_{\tau}=(\tau, x)\right\}$. Each slice $S_{\tau}$ is then considered to represent a position basis $\left\{\left|x_{\tau}\right\rangle\right\}$ which can be associated with a corresponding Hilbert space $\mathcal{H}$ with elements $\left|x_{\tau}\right\rangle$ labelled by a continuous variable $x_{\tau}$ normalised using the Dirac $\delta$-function:

$$
\left\langle x_{\tau}^{\prime} \mid x_{\tau}\right\rangle=\delta\left(x_{\tau}^{\prime}-x_{\tau}\right)
$$

The invariant unit state vector $|\varphi(\tau)\rangle$ in the Hilbert space $\mathcal{H}$ associated with slice $S_{\tau}$ can then be expanded as an integral function of the base elements $\mid x_{\tau}$ as follows:

$$
|\varphi(\tau)\rangle=\int d x_{\tau} \varphi\left(x_{\tau}\right)\left|x_{\tau}\right\rangle
$$

The right-hand side of Eq. 3.2 is defined by a line integral along the piecewise smooth curve representing slice $S_{\tau}$. It describes the state vector $|\varphi(\tau)\rangle$ of a physical system as a superposition of position basis elements $\left|x_{\tau}\right\rangle$ each one of which corresponds to a definite position $(\tau, x)$ on the slice $S_{\tau}$. The expanding coefficients or "weights" $\varphi\left(x_{\tau}\right)$ represent a complex-valued invariant wavefunction.
The left-hand side $|\varphi(\tau)\rangle$ belongs to the Hilbert space $\mathcal{H}$ and represents the vector sum or the resultant of the decomposed position states. As all the superposed arguments are defined at the same invariant proper time instant $\tau$, there is a sense of calculating their resultant.

The arguments $x_{\tau}$ of the wavefunction $\varphi\left(x_{\tau}\right)$ are associated with the points $(\tau, x)$ of the corresponding slice $S_{\tau}$. The wavefunction $\varphi\left(x_{\tau}\right)$ being defined with respect to proper time $\tau$ is relativistically invariant and shall be called "invariant wavefunction." Thus, the position state of a physical system at any given proper time $\tau$ is represented by an invariant wavefunction $\varphi(\tau, x)$ where $x \in]-\infty,+\infty[$ and has a corresponding relativistic energy $E(\tau, x)$ at that specific time $\tau$.

Hereafter, we define the evolution of the invariant wavefunction $\varphi(\tau, x)$ from one slice to a subsequent slice. A simple method is to use the relativistic energy $E(\tau, x)$ associated with the invariant wavefunction $\varphi(\tau, x)$ of a system having a definite momentum $P$ (or velocity $v$ ) with respect to an inertial frame of reference. The relativistic energy $E(\tau, x)$ is defined as follows:

$$
E=\sqrt{m^{2} c^{4}+P^{2} c^{2}}=m c^{2} / \sqrt{1-v^{2} / c^{2}}
$$

The differential quantum operator associated with energy $E$ is given by:

$$
i \hbar \partial / \partial t \equiv E
$$

It should be noted that momentum and energy operators are generators of translations in space $\mathrm{x}$ and time $\mathrm{t}$, respectively, and they operate on the wavefunction to quantify the rate of change of its states. Thus, even though the momentum and energy operators are defined as functions of differentials in space $x$ and time $t$, respectively, the corresponding momentum and energy observables should not necessarily depend explicitly on space and/or time variables. For example, the momentum and energy observables are stationary for free particles.

To define the energy operator with respect to proper time, we use Eq. 2.6 to express the differential of proper time $\delta \tau$ as a function of the differentials of ordinary time $\delta t$ and space $\delta x$ as follows:

$$
\delta \tau= \pm \sqrt{\delta t^{2}-\delta x^{2}}
$$

The "+/-" signs designate vectors inside the upper/lower light cones. In the upper light-cone $\delta \tau \geq 0$ and correspondingly $\delta t \geq 0$, whereas in the lower light cone $\delta \tau \leq 0$ and correspondingly $\delta t \leq 0$. Thus, the "+" sign designates a future-directed proper time $\delta \tau$ and correspondingly a future-directed standard time $\delta t$, while the “-” sign designates a past-directed proper time $\delta \tau$ and correspondingly a past-directed standard time $\delta t$.

Using relation (3.5), the differential $\delta \tau$ can be expressed as follows:

$$
\delta \tau= \pm \delta t \sqrt{1-\frac{(\delta x / \delta t)^{2}}{c^{2}}}= \pm \delta t \sqrt{1-v^{2} / c^{2}}
$$

However, the term $\sqrt{1-v^{2} / c^{2}}$ can be expressed as a function of the relativistic energy $E$, as 


$$
\sqrt{1-v^{2} / c^{2}}=m c^{2} / E
$$

Injecting Eq. 3.7 into Eq. 3.6, we obtain

$$
\delta \tau= \pm \delta t\left(m c^{2} / E\right)
$$

By substituting Eq. 3.8 into Eq. 3.4, we obtain the following energy operator with respect to the proper time:

$$
i \hbar \partial / \partial \tau \equiv \pm E^{2} / m c^{2} \equiv \pm \mathcal{E}
$$

The left-hand term of the above expression (17) is the differential operator with respect to proper time $\tau$ and the middle term represents an associated energy which shall be called "proper energy operator" and is symbolised in the right-hand term by $\mathcal{E}$ which can be considered as a proper-time evolution operator.

Thus, to describe the evolution of the invariant wavefunction $\varphi(\tau, x)$ with respect to the proper time, we apply the above operator (3.9) as follows:

$$
i \hbar \frac{\partial \varphi(\tau, x)}{\partial \tau}= \pm\left(E^{2} / m c^{2}\right) \varphi(\tau, x)= \pm \mathcal{E} \varphi(\tau, x)
$$

The solutions of the above system of equations are:

$$
\varphi(\tau, x)=\varphi_{0} e^{ \pm \mathrm{i}\left(E^{2} / \hbar m c^{2}\right) \tau} \equiv \varphi_{0} e^{ \pm \mathrm{i}(\mathcal{E} / \hbar) \tau}
$$

Where $\varphi_{0}$ is an initial distribution.

The above solutions can be considered as invariant plane waves with always positive energies. These solutions show that for example, in the future-directed dynamics, an increase in proper time $\tau$ changes the phase of the proper wavefunction at a proper-time rate $E^{2} / \hbar m c^{2}$.

Equation 3.11 implies that the proper-time evolution operator on an isolated system can be described by unitary operators $\mathscr{W}$ defined as follows:

$$
\mathscr{U}(\tau) \equiv e^{ \pm \mathrm{i}\left(E^{2} / \hbar m c^{2}\right) \tau}
$$

Where the "+” sign (respectively, “-” sign) designates a futuredirected (respectively, past-directed) evolution of the invariant wavefunction $\varphi(\tau, x)$ with respect to proper time $\tau$.

Expanding expression (3.10) into a system of two equations, while using the relativistic energy $E$ of Eq. 3.3, we obtain

$$
\left\{\begin{array}{cl}
i \hbar \frac{\partial \varphi(\tau, x)}{\partial \tau}=+\left(m c^{2}+P^{2} / m\right) \varphi(\tau, x) & \text { future-directed } \\
i \hbar \frac{\partial \varphi(\tau, x)}{\partial \tau}=-\left(m c^{2}+P^{2} / m\right) \varphi(\tau, x) & \text { past-directed }
\end{array}\right.
$$

Expressing the momentum $P$ according to its corresponding operator $-i \hbar \partial / \partial x$, the above set of Eq. 3.13 becomes

$$
\begin{cases}i \hbar \frac{\partial \varphi(\tau, x)}{\partial \tau}=+\left(m c^{2}-\frac{\hbar^{2}}{m} \frac{\partial^{2}}{\partial x^{2}}\right) \varphi(\tau, x) & \text { future-directed } \\ i \hbar \frac{\partial \varphi(\tau, x)}{\partial \tau}=-\left(m c^{2}-\frac{\hbar^{2}}{m} \frac{\partial^{2}}{\partial x^{2}}\right) \varphi(\tau, x) & \text { past-directed }\end{cases}
$$

Eq. 3.14 are related in a simple manner to the proper time evolution. This system (Eq. 3.14) of equations shall be called "invariant equation of motion" describing the evolution of the invariant wavefunction $\varphi(\tau, x)$. It is composed of two different dynamics: the first equation corresponds to a futuredirected dynamics taking place within a future-light cone, while the second equation corresponds to a past-directed dynamics taking place within a past-light cone. Each equation is in the conventional sense, that is the time reversal of the other. That is, flipping the direction of proper time $(\tau \rightarrow-\tau)$ in any one of Eq. 3.14, leads to the other one of these equations.

The system of invariant equations of motion (3.14) can be considered a relativistic Schrodinger equation of motion. Indeed, by introducing Eq. 3.8 into the system of Eq. 3.10 while replacing the invariant wavefunction $\varphi(\tau, x)$ by the standard wavefunction $\psi(x, t)$, we obtain

$$
\left\{\begin{array}{cc}
i \hbar \frac{\partial \psi(x, t)}{\partial t}=+E \psi(x, t) & \text { futur-directed } \\
i \hbar \frac{\partial \psi(x, t)}{\partial t}=-E \psi(x, t) & \text { past-directed }
\end{array}\right.
$$

The energy $E$ for a free particle in the non-relativistic limit can be expressed as follows:

$$
E=\sqrt{m^{2} c^{4}+P^{2} c^{2}}=m c^{2} \sqrt{1+v^{2} / c^{2}} \approx m c^{2}+m v^{2} / 2
$$

Introducing the approximation of Eq. 3.16 into the above system of Eq. 3.15, we obtain

$$
\begin{cases}i \hbar \frac{\partial \psi(x, t)}{\partial t}=\left(m c^{2}+m v^{2} / 2\right) \psi(x, t) & \text { for } t \geq 0 \\ i \hbar \frac{\partial \psi(x, t)}{\partial t}=-\left(m c^{2}+m v^{2} / 2\right) \psi(x, t) & \text { for } t \leq 0\end{cases}
$$

We note that the term $m c^{2}$ is a constant corresponding to the rest energy that has no consequences for the evolution of the physical system and can be omitted. Moreover, the nonrelativistic kinetic energy $m v^{2} / 2$ can be expressed as $p^{2} / 2 m$, leading to the following system of equations:

$$
\begin{cases}i \hbar \frac{\partial \psi(x, t)}{\partial t}=\left(p^{2} / 2 m\right) \psi(x, t) & \text { for } t \geq 0 \\ i \hbar \frac{\partial \psi(x, t)}{\partial t}=-\left(p^{2} / 2 m\right) \psi(x, t) & \text { for } t \leq 0\end{cases}
$$

The first equation corresponds to Schrodinger's standard equation for a free particle in the future direction, while the second equation corresponds to Schrodinger's equation in the past direction.

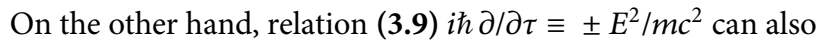
be expressed as a product of two terms:

$$
\left(i \hbar \frac{\partial}{\partial \tau}+E^{2} / m c^{2}\right)\left(i \hbar \frac{\partial}{\partial \tau}-E^{2} / m c^{2}\right) \equiv 0
$$

Thus, applying the above operator to the invariant wavefunction $\varphi(\tau, x)$ with respect to the proper time 


$$
\left(i \hbar \frac{\partial}{\partial \tau}+E^{2} / m c^{2}\right)\left(i \hbar \frac{\partial}{\partial \tau}-E^{2} / m c^{2}\right) \varphi(\tau, x)=0
$$

Developing Eq. 3.20, we obtain an invariant equation of motion equivalent to the system of Eq. 3.10, as follows:

$$
\left(-\hbar^{2} \frac{\partial^{2}}{\partial \tau^{2}}-\left(E^{2} / m c^{2}\right)^{2}\right) \varphi(\tau, x)=0
$$

On the other hand, squaring expression (3.8), one gets:

$$
\delta \tau^{2}=\delta t^{2}\left(m c^{2} / E\right)^{2}
$$

Then, using the above relation, Eq. 3.21 can be transformed into

$$
\left(-\hbar^{2} \frac{\partial^{2}}{\partial t^{2}}-E^{2}\right) \varphi(\tau, x)=0
$$

Introducing the identity of relativistic energy (3.3) into Eq. 3.23, the latter becomes

$$
\left(-\hbar^{2} \frac{\partial^{2}}{\partial t^{2}}-m^{2} c^{4}-P^{2} c^{2}\right) \varphi(\tau, x)=0
$$

Finally, expressing the momentum according to its corresponding operator, Eq. $\mathbf{3 . 2 4}$ becomes Klein-Gordon's equation:

$$
-\hbar^{2} \frac{\partial^{2} \psi(x, t)}{\partial t^{2}}=\left(-\hbar^{2} c^{2} \nabla^{2}+m^{2} c^{4}\right) \varphi(\tau, x)
$$

The above analysis shows that the dynamics of the invariant wavefunction with respect to proper time is, on the one hand, equivalent to Klein-Gordon's equation, and on the other hand, yields Schrodinger's equation in the non-relativistic limit. This clearly confirms that the original hypothesis of defining the wavefunction with respect to the proper time is reasonable.

On the other hand, the first and second Eqs 3.18 are not mirror images of each other. However, flipping the direction of time $(t \rightarrow-t)$ in any one of Eqs 3.18, we obtain the other one of these equations. Thus, when the system of equations is taken as a whole, time reversal consists merely of turning time's sign around in the same way as it is defined in classical mechanics.

Nevertheless, each equation is invariant under the standard quantum mechanics time-reversal operator [28] within its corresponding light cone. That is, a time-reversal anti-unitary operator consisting of changing the sign of time $(t \rightarrow-t)$ and taking the complex conjugate is exclusively applicable either in the upper light cone or in the lower light cone. This transformation can be interpreted as a reverse in the direction of movement and not a reverse in the direction of time. We note that some authors [29-31] have shed some doubt on the standard definition of time reversal in quantum mechanics.

\section{ONTOLOGY OF THE INVARIANT WAVEFUNCTION}

To gain insight into the ontology of the invariant wavefunction, it would be appropriate to derive the continuity equation from the invariant system of equations of motion (3.14). Hereafter, the continuity equation is considered only in the future-directed upper cone. Multiplying the first equation by the conjugate invariant wavefunction $\varphi^{*}(\tau, x)$ and multiplying the complex conjugate of Eq. 3.14a by the invariant wavefunction $\varphi(\tau, x)$, gives

$$
\begin{aligned}
i \hbar \varphi^{*} \frac{\partial \varphi}{\partial \tau} & =+\varphi^{*}\left(m c^{2}-\frac{\hbar^{2}}{m} \frac{\partial^{2}}{\partial x^{2}}\right) \varphi \\
-i \hbar \varphi \frac{\partial \varphi^{*}}{\partial \tau} & =+\varphi\left(m c^{2}-\frac{\hbar^{2}}{m} \frac{\partial^{2}}{\partial x^{2}}\right) \varphi^{*}
\end{aligned}
$$

Subtracting Eq. 4.2 from the first Eq. 4.1 yields the following result:

$$
i \hbar \frac{\partial \varphi^{*} \varphi}{\partial \tau}=\frac{\hbar^{2}}{m}\left(\varphi \frac{\partial^{2} \varphi^{*}}{\partial x^{2}}-\varphi^{*} \frac{\partial^{2} \varphi}{\partial x^{2}}\right)
$$

The above Eq. 4.3 can be simplified, as follows:

$$
\frac{\partial|\varphi|^{2}}{\partial \tau}=-\frac{i \hbar}{m} \frac{\partial}{\partial x}\left(\varphi \frac{\partial \varphi^{*}}{\partial x}-\varphi^{*} \frac{\partial \varphi}{\partial x}\right)
$$

Equation 4.4 can be written as a continuity equation:

$$
\frac{\partial \rho}{\partial \tau}+\frac{\partial}{\partial x} j=0
$$

Where the current $j$ and the density $\rho$ are given by:

$$
\begin{gathered}
j=-\frac{i \hbar}{m}\left(\varphi \frac{\partial \varphi^{*}}{\partial x}-\varphi^{*} \frac{\partial \varphi}{\partial x}\right) \\
\rho \equiv \rho\left(x_{\tau}\right)=|\varphi(\tau, x)|^{2} \equiv|\varphi|^{2}
\end{gathered}
$$

More generally, in a three-dimensional space $(x, y, z)$, the current $\vec{j}$ can be expressed as follows:

$$
\vec{j}=-\frac{i \hbar}{m}\left(\varphi \vec{\nabla} \varphi^{*}-\varphi^{*} \vec{\nabla} \varphi\right)
$$

Then, the three-dimensional continuity equation becomes:

$$
\frac{\partial \rho}{\partial \tau}+\vec{\nabla} \cdot \vec{j}=0
$$

Integrating the continuity equation over the volume of the entire space:

$$
\frac{d}{d \tau} \int \rho d x d y d z=\int \frac{\partial \rho}{\partial \tau} d x d y d z=-\int \vec{\nabla} \cdot \vec{j} d x d y d z=\int \vec{j} d^{2} s
$$

In the last equality, Gauss's theorem is used to transform the volume integral into a surface integral over $s$. The last integral is equal to zero, as the current $\vec{j}$ vanishes at the boundary of surface $s$ at infinity. Thus, Eq. $\mathbf{4 . 1 0}$ becomes

$$
\frac{d}{d \tau} \int \rho d x d y d z=0
$$

Therefore, the integral of $\rho=|\varphi|^{2}$ over the entire space is conserved with respect to the proper time $\tau$. 
It can be outlined from the preceding sections that an invariant wavefunction $\varphi\left(x_{\tau}\right) \equiv \varphi(\tau, x)$ has two main properties. The first property is that all the arguments of the invariant wavefunction are associated with the points $(\tau, x)$ of a corresponding slice $S_{\tau}$, and are thus defined at a specific proper time instant $\tau$ which is invariant for all observers. The second property is that the evolution of the invariant wavefunction through proper time is unitary; that is, its global density $\rho=|\varphi(\tau)|^{2}$ is conserved at each proper time instant $\tau$ regardless of the spacetime interval between any two arguments. These first and second properties imply that the invariant wavefunction forms a holistic bloc with an inseparable identity. The invariant wavefunction $\varphi(\tau, x)$ can thus be considered as a single "quantum event," which describes the quantum state of a physical system at a specific proper time $\tau$.

Moreover, as explained by Tim Maudlin in his book "Philosophy of Physics: Quantum Theory [32], the wavefunction accounts faithfully for the interference pattern (for example, in a two-slit experiment), and it is reasonable to consider that it represents some real physical features of a physical system. The characteristics and behaviour of the wavefunction should reflect those of the physical system.

Indeed, Schrodinger originally suggested that a particle can be assimilated to a "cloud" that continuously fills the entire space and whose density is given by the square of the wavefunction. This idea has been abandoned by Schrodinger mainly because the cloud continues to diffuse and does not seem to correspond to the relatively sharp macroscopic world [1]. Moreover, all the points of the cloud are defined at the same instant of time, thus contradicting the relative simultaneity principle of special relativity.

Nevertheless, this idea was reintroduced in GRW theory [1, 14]. Hereafter, we adopt this concept in relation to the invariant wavefunction whose points are defined at the same proper time instant which is invariant for all observers and thus, does not contradict the principles of special relativity. The diffusion of Schrodinger's cloud is highlighted by the continuity Eq. 4.9.

The physical system may thus be considered as a type of continuously spread cloud that we shall call "individual fabric", which makes part of the spacetime fabric itself. Each individual fabric has its proper identity (or individuality) and has a finite "density amplitude" that vanishes at infinity, reflecting the effects and properties of the corresponding invariant wavefunction. In particular, the density $\rho\left(x_{\tau}\right)$ of the individual fabric at each point $x_{\tau}$ is equal to the square of the invariant wavefunction at that point, as expressed by Eq. 4.7.

The dispersion and probabilities of the invariant wavefunction reflect the real density distribution within the corresponding individual fabric. For example, an invariant wavefunction whose density amplitude is defined by a normal distribution would be the result of a normal distribution of the density of the individual fabric. In particular, the dispersion of the invariant wavefunction represents the width of the central region where the density of the individual fabric is concentrated, and the median is the centre of density of the individual fabric, while the tails would represent rarefied peripheral density regions. The region where the density is concentrated interacts the most with its environment and is called the "useful part" of the individual fabric, whereas the rarefied density regions have little or no interactions with their environment.

The idea that the invariant wavefunction $\varphi(\tau, x)$ represents an individual fabric as a single bloc or a single quantum event can be best explained by the notion of velocity. In particular, the individual fabric can be represented by its centre of density $\mathrm{X}(\tau)$. It should be noted that the centre of density $\mathrm{X}(\tau)$ is only a simple manner to represent the distribution of density within a fabric akin to the centre of mass in classical physics and is not a hidden variable.

We consider an individual fabric represented by an invariant wavefunction $\varphi(\tau, x, y, z)$ that can be expressed as a function of its amplitude $R(\tau, x, y, z)$ and phase $S(\tau, x, y, z)$ as follows:

$$
\varphi(\tau, x, y, z)=R(\tau, x, y, z) e^{i S(\tau, x, y, z)}
$$

To express the notion of velocity, we introduce the above formulation into the current Eq. 4.8:

$$
\vec{j}=\frac{2 \hbar}{m} R^{2} \vec{\nabla} S=\frac{2 \hbar}{m} \rho \vec{\nabla} S
$$

The current $\vec{j}$ can be expressed as the density multiplied by the velocity $\vec{U}$ and Thus, in a manner similar to the de Broglie Bohm theorem, the velocity $\vec{U}$ can be related to the phase $S(\tau, x, y, z)$ of the wavefunction, according to the following equation:

$$
\vec{U}=\left(\frac{d x}{d \tau} ; \frac{d y}{d \tau} ; \frac{d z}{d \tau}\right)=\left.\frac{2 \hbar}{m} \vec{\nabla} S\right|_{X(\tau)}
$$

The gradient of the phase $\vec{\nabla} S$ is evaluated at the location of the individual fabric's centre of density $X(\tau)$. Here, $\vec{U}$ is an invariant velocity corresponding to the space components of the 4relativistic velocity. The invariant velocity $\vec{U}$ represents the invariant velocity of the centre of density $\mathrm{X}(\tau)$.

The difference with de Broglie Bohm's theorem is that here, the parameter $\mathrm{X}(\tau)$ does not represent the actual position of a particle but simply a centre of density of the fabric represented by the wavefunction. This centre of density, $\mathrm{X}(\tau)$ is not necessarily located in the densest region of the fabric. For example, in the case of a wavefunction moving across two slits, the dense region is distributed between two zones corresponding to the locations of these two slits, and thus, the centre of density is somewhere in between. Consequently, the zone of interaction during a measurement is not necessarily where the centre of density $\mathrm{X}(\tau)$ is located.

For a free particle, the centre of density $\mathrm{X}(\tau)$ of the individual fabric may be interpreted as the centre of a sphere-like space-time fabric whose density diminishes at infinity. The centre of density $\mathrm{X}(\tau)$ can be assimilated to a point particle, as in point mechanics.

In general, the invariant wavefunction $\varphi(\vec{x}, \tau) \equiv \varphi\left(\vec{x}_{1}, \ldots, \vec{x}_{i}, \ldots \vec{x}_{k}, \tau\right)$ of a set of physical systems composed of $\mathrm{k}$ particles is defined in a high-dimensional configuration space on $\mathrm{R}^{3 \mathrm{k}} \times \mathrm{R}$, codifying their different positions and identities, where $\vec{x} \equiv\left(\vec{x}_{1}, \ldots, \vec{x}_{i}, \ldots \vec{x}_{k}\right)$ and $\vec{x}_{i} \equiv\left(x_{i}, y_{i}, z_{i}\right)$. 
Analogous to the expression disclosed in Norsen [1], the density distribution $\rho_{i}\left(\tau, \vec{x}_{i}\right)$ of the $i$ th individual fabric is the integral of the square of the invariant wavefunction $|\varphi(\vec{x}, \tau)|^{2}$ over the coordinates associated with all the other individual fabrics, as follows:

$$
\rho_{i}\left(\tau, \vec{x}_{i}\right)=\int|\varphi|^{2} \delta^{3}\left(\vec{x}_{i}-\vec{x}\right) d^{3} x_{1} d^{3} x_{2} \ldots d^{3} x_{k}
$$

The above expression gives the density distribution $\rho_{i}\left(\tau, \vec{x}_{i}\right)$ of the $i$ th individual fabric by integrating over the coordinates associated with all the other individual fabrics. The result $\rho_{i}\left(\tau, \vec{x}_{i}\right) \equiv \rho_{i}\left(\tau, x_{i}, y_{i}, z_{i}\right)$ is thus defined as a function of the proper time $\tau$ and only three space coordinates $\left(x_{i}, y_{i}, z_{i}\right)$.

Then, the global density $\rho(\tau, \vec{x})$ of the global fabric representing the $\mathrm{k}$-particle-system is the sum of all the individual densities $\rho_{i}\left(\tau, \vec{x}_{1}\right), \ldots, \rho_{i}\left(\tau, \vec{x}_{i}\right), \ldots, \rho_{i}\left(\tau, \vec{x}_{k}\right)$ of the corresponding individual fabrics representing the $\mathrm{k}$ different particles, as follows:

$$
\rho(\tau, \vec{x})=\sum_{i=1}^{k} \rho_{i}\left(\tau, \vec{x}_{i}\right)
$$

Where each individual density $\rho_{i}\left(\tau, \vec{x}_{i}\right) \equiv \rho_{i}\left(\tau, x_{i}, y_{i}, z_{i}\right)$ is defined as a function of proper time $\tau$ and only three space coordinates $\left(x_{i}, y_{i}, z_{i}\right)$ which can be simply identified with the three coordinates $(x, y, z)$ of the physical space. Thus, the global density $\rho(\tau, \vec{x})$ can be reformulated as follows:

$$
\rho(\tau, x, y, z)=\sum_{i=1}^{k} \rho_{i}(\tau, x, y, z)
$$

This extrapolates Schrodinger's original idea by first defining the individual density for each particle separately, and then by constructing the global density as the "sum" of all individual densities. Thus, each particle corresponds to an individual fabric whose points $(\tau, x, y, z)$ are defined in a four-dimensional physical space time $\mathrm{R} \times \mathrm{R}^{3}$ such that the density distribution of each individual fabric continuously fills the entire space. All individual fabrics are isomorphic, and their density distributions are defined on the same four-dimensional physical space time $\mathrm{R} \times \mathrm{R}^{3}$.

Consequently, the multi-particle invariant wavefunction $\varphi\left(\vec{x}_{1}, \ldots, \vec{x}_{i}, \ldots \vec{x}_{k}, \tau\right)$ can be perceived as representing a "global fabric" defined in the four-dimensional physical space time $\mathrm{R} \times \mathrm{R}^{3}$ and whose density distribution corresponds to the sum of all the density distributions of its constituent individual fabrics. In other words, the global fabric is defined by a global density distribution $\rho(\tau, x, y, z)$ over a set of points $(\tau, x, y, z)$ in a four-dimensional spacetime $\mathrm{R} \times \mathrm{R}^{3}$ where each point represents a common position of all the $\mathrm{k}$-individual positions. Moreover, we note that here, the k-particle system is unambiguously defined at a specific proper time instant $\tau$ regardless of the distance between the different centres of density and does not contradict relativity.

However, the global density distribution $\rho(\tau, x, y, z)$ provides only a partial representation of the global fabric. Indeed, by making the above summing operation, we lose the individual identity of each individual fabric, knowing that these individual identities also determine the way they interact with each other to form the global fabric. This can be illustrated by breaking the invariant wavefunction $\varphi(\vec{x}, \tau) \equiv \varphi\left(\vec{x}_{1}, \ldots, \vec{x}_{i}, \ldots \vec{x}_{k}, \tau\right)$ into its amplitude $R(\vec{x}, \tau)$ and phase $S(\vec{x}, \tau)$, as follows:

$$
\varphi(\vec{x}, \tau)=R(\vec{x}, \tau) e^{i S(\vec{x}, \tau)} \equiv \sqrt{\rho(\tau, x, y, z)} e^{i S(\vec{x}, \tau)}
$$

The amplitude is expressed as a function of the global density distribution $\rho(\tau, x, y, z)$ defined in physical space; thus, the information concerning the individual fabrics and their interrelations should be encoded in the phase $S(\vec{x}, \tau) \equiv S\left(\vec{x}_{1}, \ldots, \vec{x}_{i}, \ldots \vec{x}_{k}, \tau\right)$.

Generalising the above velocity expression, the velocity $\vec{U} \equiv\left(\vec{U}_{1}, \ldots, \vec{U}_{i}, \ldots \vec{U}_{k}\right)$ relative to a plurality of individual fabrics, is given by the following:

$$
\vec{U} \equiv\left(\vec{U}_{1}, \ldots, \vec{U}_{i}, \ldots \vec{U}_{k}\right)=\frac{2 \hbar}{m}\left(\left.\vec{\nabla} S\right|_{X_{1}}, \ldots,\left.\vec{\nabla} S\right|_{X_{i}},\left.\ldots \vec{\nabla} S\right|_{X_{k}}\right)
$$

Where each $\vec{U}_{i}$ represents the invariant velocity of the centre of density $X_{i}(t)$ relative to the $i$ th individual fabric. The invariant velocity $\vec{U}_{i}$ is expressed as follows:

$$
\vec{U}_{i}=\frac{d}{d \tau} X_{i}(\tau)=\left.\frac{2 \hbar}{m} \vec{\nabla} S\left(\vec{X}_{1}, \ldots, \vec{x}_{i}, \ldots \vec{X}_{k}, \tau\right)\right|_{x_{i}=X_{i}}
$$

The gradient of the phase $\vec{\nabla} S$ is evaluated at the location of the $i$ th individual fabric's centre of density $X_{i}(t)$. However, the variation in the centre of density of one individual fabric affects all other individual fabrics if they are correlated and has no effect if non-correlated.

Indeed, if the particles are correlated, then any change $\delta X_{j}$ in the centre of density $X_{j}$ of the $j$ th individual fabric changes the value of the phase $S\left(\vec{X}_{1}, \ldots, \vec{x}_{i}, \ldots, \vec{X}_{j}+\delta \vec{X}_{j}, \ldots \vec{X}_{k}, \tau\right)$ which automatically changes the centre of density $X_{i}$ of the $i$ th individual fabric according to Eq. 4.20. This variation in the centre of density describes a non-local inherent transformation within the individual fabric and does not imply any displacement of the individual fabric.

However, if the particles are not correlated, the wavefunction $\varphi(\vec{x}, \tau) \equiv \varphi\left(\vec{x}_{1}, \ldots, \vec{x}_{i}, \ldots \vec{x}_{k}, \tau\right)$ factors into a product of independent wavefunctions:

$$
\begin{aligned}
\varphi(\vec{x}, \tau) & =\varphi\left(\vec{x}_{1}, \ldots, \vec{x}_{i}, \ldots \vec{x}_{k}, \tau\right) \\
& =\varphi\left(\vec{x}_{1}, \tau\right) \ldots \varphi\left(\vec{x}_{i}, \tau\right) \ldots \varphi\left(\vec{x}_{k}, \tau\right)
\end{aligned}
$$

The above relation (4.21) can also be expressed in polar form:

$$
\begin{aligned}
\varphi\left(\vec{x}_{1}, \ldots, \vec{x}_{k}, \tau\right) & =R\left(\vec{x}_{1}, \ldots, \vec{x}_{k}, \tau\right) e^{i S\left(\vec{x}_{1}, \ldots, \vec{x}_{k}, \tau\right)} \\
& =R\left(\vec{x}_{1}, \tau\right) e^{i S\left(\vec{x}_{1}, \tau\right)} \ldots R\left(\vec{x}_{k}, \tau\right) e^{i S\left(\vec{x}_{k}, \tau\right)}
\end{aligned}
$$

Such that;

$$
\begin{gathered}
S\left(\vec{x}_{1}, \ldots, \vec{x}_{i}, \ldots \vec{x}_{k}, \tau\right)=\sum_{i=1}^{k} S\left(\vec{x}_{i}, \tau\right) \\
R\left(\vec{x}_{1}, \ldots, \vec{x}_{k}, \tau\right)=\prod_{i=1}^{k} R_{i}\left(x_{i}, t\right)
\end{gathered}
$$


In this case, for any $i$, the velocity $\vec{U}_{i}$ depends only on the phase relative to its own individual fabric, as follows:

$$
\vec{U}_{i}=\frac{d}{d \tau} X_{i}(\tau)=\left.\frac{2 \hbar}{m} \vec{\nabla} S\left(\vec{x}_{i}, \tau\right)\right|_{x_{i}=X_{i}}
$$

In view of the above, it can be concluded that the global fabric should be represented by the multi-particle invariant wavefunction $\varphi\left(\vec{x}_{1}, \ldots, \vec{x}_{i}, \ldots \vec{x}_{k}, \tau\right)$ which is an abstract mathematical representation that defines the individual identities of all individual fabrics as well as, though indirectly, the global density $\rho(\tau, x, y, z)$ as derived above. In other words, the multi-particle invariant wavefunction $\varphi\left(\vec{x}_{1}, \ldots, \vec{x}_{i}, \ldots \vec{x}_{k}, \tau\right)$ provides a consistent definition of the global fabric as it determines the global density $\rho(\tau, x, y, z)$ according to Schrodinger's summing operation on all individual fabric densities while conserving their individual identities.

Moreover, as seen above, the multi-particle invariant wavefunction $\varphi\left(\vec{x}_{1}, \ldots, \vec{x}_{i}, \ldots \vec{x}_{k}, \tau\right)$ determines whether the merging according to the above Schrodinger's summing operation is a simple "juxtaposition" or a "combination" of the different density distributions.

A non-factorable invariant wavefunction means that the individual fabrics are "combined" together; that is, they are intermingled or entangled with each other as if formed of a single fabric (having a common global identity) representing a single quantum event. On the other hand, a factorable invariant wavefunction means that the individual fabrics are only "juxtaposed" to each other without being entangled, representing simultaneous quantum events with respect to the proper time.

\section{CONTRACTING FUNCTION}

The invariant wavefunction $\varphi(\tau, x)$ representing a free particle corresponds to an individual fabric that evolves according to the invariant equation of motion (3.14) as an indivisible holistic bloc within the upper (or lower) light cone; thus, its evolution is local and consistent with special relativity. During this evolution, the invariant wavefunction spreads out in the standard space in a smooth manner as a consequence of its passage from one slice $S_{\tau_{1}}$ into a subsequent slice $S_{\tau_{2}}$. This spreading out suggests that the individual fabric's density should be stretched out (i.e. becoming more diluted) while evolving through proper time.

However, the introduction of an external potential such as a confining potential $V(\tau, x)$ modifies the form of the invariant equation of motion (15). The confining potential $V(\tau, x)$ acts on the useful part of the individual fabric which in turn reacts by undergoing an abrupt redistribution and, more precisely, an abrupt contraction of its density in compliance with the newly introduced potential.

The abrupt redistribution of the individual fabric's density due to the abrupt introduction of the external potential may be formalised by multiplying the invariant wavefunction by a contracting function $\gamma(\tau, x)$ whose form depends on the external potential, as follows:

$$
\varphi^{+}(\tau, x)=\gamma(\tau, x) \varphi^{-}(\tau, x)
$$

Where $\varphi^{+}(\tau, x)$ and $\varphi^{-}(\tau, x)$ represent the subsequent and antecedent invariant wavefunctions for all observers, respectively. Indeed, the proper time instant $\tau$ is invariant for all observers, and it is the instant at which the external potential $V(\tau, x)$ acts on the antecedent wavefunction $\varphi^{-}(\tau, x)$ to instantaneously produce the subsequent wavefunction $\varphi^{+}(\tau, x)$.

Equation 5.1 implies that at a proper time instant $\tau-\delta \tau$, just before the action of the external potential $V(\tau, x)$, the invariant wavefunction is in an antecedent state $\varphi^{-}(\tau-\delta \tau, x)$ and that a proper time instant $\tau+\delta \tau$, just after the action of the external potential $V(\tau, x)$, the invariant wavefunction is in a subsequent state $\varphi^{+}(\tau+\delta \tau, x)$.

In general, the contracting function $\gamma(\tau, x)$ can be defined by a contracting Gaussian $\mathrm{g}(\tau, x)$ applied to the zone of interaction of the invariant wavefunction and whose dispersion depends on the form of the external confining potential. The narrower the confinement potential, the smaller the wavefunction dispersion, and thus, the more concentrated the dense region of the fabric.

However, in accordance with the uncertainty principle, the density of the individual fabric cannot be concentrated beyond an infinitesimally small minimal localised region. In the latter case, the contracting Gaussian $\mathrm{g}(\tau, x)$ becomes a localising Gaussian $\mathrm{g}_{\mathrm{X}}(\tau, x)$ similar to that in the GRW theory, except that it is not a random spontaneous collapse but rather a deterministic response to the external potential, as will be described in more detail in Measurement, Discussion. The localising Gaussian $\mathrm{g}_{\mathrm{X}}(\tau, x)$ has a minimal dispersion $\varepsilon$ consistent with the uncertainty principle and is centred at a point $\mathrm{x}_{\tau}=(\tau, X)$ around which the contraction takes place. Thus, unlike the GRW theory, the point $(\tau, X)$ is not random and depends on the region of interaction between the wavefunction and the external potential that triggers the contraction. On the other hand, point $(\tau, X)$ is not necessarily the centre of density.

\section{APPLICATION: EVOLUTION OF A PARTICLE THROUGH A SLIT}

Let a particle exit from a source, travel towards a barrier with a slit, and pass through the slit before impacting the screen. Let the source be at the origin $O$ of a standard spatial coordinate system $(O ; x, y, z)$ such that the particle travels in the $y$-direction toward the barrier, the screen and the barrier being both parallel to the $(x, z)$ plane, and the barrier slit is along the $\mathrm{z}$-direction. For simplicity, we neglect the $\mathrm{z}$-direction and restrict the analysis to a two-dimensional space with respect to system $(O ; x, y)$.

A family of slices $S_{\tau}(\tau, x, y)$ composed of two-dimensional hyperboloids are generated by "proper time vectors" $\vec{\tau}$ arising from the origin $O$. This family of slices $\left(S_{\tau}\right)_{\tau \in R}$ forms a threedimensional spacetime coordinate system $(O ; \tau, x, y)$ in which the evolution of an invariant wavefunction representing the free particle can be studied.

The evolution of the particle through the proper time $\tau$ is decomposed into three periods. The first period was from the 
source to the barrier, a second period through the slit, and a third period from the slit to the screen.

\section{First Period From the Source to the Barrier}

Suppose that initially, at $\tau=0$, just downstream from the source, the particle corresponds to a free particle represented by a Gaussian wavefunction. In particular, the wavefunction's transverse profile (i.e. in the $\mathrm{x}$-direction) can be defined by a Gaussian with a dispersion $\sigma$, as follows:

$$
\varphi(0, x)=\frac{1}{\left(2 \pi \sigma^{2}\right)^{1 / 4}} e^{-\frac{x^{2}}{4 \sigma^{2}}}
$$

At a later proper time instant $\tau$ and by using the same analysis as that of the standard wavefunction's transverse profile $[1,18]$, the transverse profile of the invariant wavefunction $\varphi(\tau, x)$ can be expressed by the following Gaussian wavefunction:

$$
\varphi(\tau, x) \approx A(\tau) e^{-\frac{x^{2}}{4 \sigma^{2}(\tau)}}
$$

Where $A(\tau)$ is the amplitude, $\sigma(\tau)$ is the dispersion, and both depend on the proper time $\tau$.

\section{Second Period at the Barrier and Through the Slit}

Suppose that the invariant wavefunction of the free particle interacts with the barrier at a certain proper time instant $\tau_{s}$. The slit potential can be regarded as an abrupt square potential, whose transverse profile is defined as follows:

$$
V(x)=\left\{\begin{array}{cc}
\varepsilon \sim 0 & \text { for }|x|<a \\
V_{0} & \text { otherwise }
\end{array}\right.
$$

Where $a$ is the width of the slit, and $V_{0} \gg 1$. That is, the slit potential is almost equal to zero within a small diameter $a$ and almost infinite elsewhere.

The slit potential acts as a contracting function $\gamma(x)$ at the proper time instant $\tau_{s}$, concentrating the density of the wavefunction within the width of the slit and diluting it outside the slit. To be consistent with the principle that a wavefunction should vanish at the edges of regions of infinite potential energy [33], the transverse contracting function $\gamma(x)$ should be a quasi-symmetrical image $S(V)$ of the transverse potential $V(x)$ with a normalising factor $1 / M$, defined as follows:

$$
\gamma(x) \sim\left\{\begin{array}{cc}
V_{0} / M & \text { for }|x|<a \\
\varepsilon / M & \text { for }|x|>a
\end{array}\right.
$$

On the other hand, to avoid the discontinuity at the sharp edges (i.e. at $|x|=a$ ), the transverse contracting function $\gamma(x)$ can be approximated by a transverse normalised contracting Gaussian $g(x)$ centred at $x=0$, with a dispersion equal to the slit width $a$, and with a normalising factor $G$ :

$$
g(x) \approx G e^{-\frac{x^{2}}{4 a^{2}}}
$$

Suppose that the useful part (i.e. dense region) of the individual fabric comes into contact with the slit barrier at an instant $\tau_{s}$, where according to Eq. 6.2, the transverse profile of its wavefunction is defined by a normalised Gaussian $\varphi^{-}\left(\tau_{s}, x, y\right)$ having a dispersion $\sigma$ much larger than the slit width $a$, as follows:

$$
\varphi^{-}\left(\tau_{s}, x, y\right)=A\left(t_{s}\right) e^{-\frac{x^{2}}{4 \sigma^{2}\left(\tau_{s}\right)}}
$$

The interaction of the individual fabric with the slit barrier instantaneously transforms the normalised Gaussian $\varphi^{-}\left(\tau_{s}, x, y\right)$ into another normalised Gaussian $\varphi^{+}\left(\tau_{s}, x, y\right)$ presenting a dispersion equal to the slit width $a$, as follows:

$$
\begin{aligned}
\varphi^{+}\left(\tau_{s}, x, y\right) & =g(x) \varphi^{-}\left(\tau_{s}, x, y\right) \\
& =G e^{-\frac{x^{2}}{4 a^{2}}} A\left(\tau_{s}\right) e^{-\frac{x^{2}}{4 \sigma^{2}\left(\tau_{s}\right)}} \approx B\left(\tau_{s}\right) e^{-\frac{x^{2}}{4 a^{2}\left(\tau_{s}\right)}}
\end{aligned}
$$

Where $B\left(\tau_{s}\right)=G \times A\left(\tau_{s}\right)$ and $\sigma \gg a$.

The multiplication of the invariant wavefunction $\varphi^{-}\left(\tau_{s}, x, y\right)$ by the normalised contracting Gaussian $g(x)$ concentrates most of its density within a certain region whose extension depends on the barrier potential. Indeed, when we describe, for example, a particle in a box with a square potential or a harmonic oscillator representing a particle in a quadratic potential, we consider that the wavefunction vanishes at the edges. In other words, we implicitly multiply the wavefunction of a free particle by a contracting function that limits its extension to a region within the barrier potential. Moreover, if the width of the box continues to decrease, the contracting function limits the dispersion of the wavefunction until it reaches an infinitesimally small minimal localised region.

However, it should be noted that the entire individual fabric represented by the invariant wavefunction crosses the slit barrier. The useful part of the individual fabric passes through the slit, while the remaining part is so rarified that it does not interact with its environment; thus, it passes through the barrier itself.

\section{Third Period From the Slit to the Screen}

Downstream from the slit, the invariant wavefunction starts at a proper time instant $\tau_{d}$, as in the first step by having a Gaussian transverse profile given by

$$
\varphi\left(\tau_{d}, x\right)=B\left(\tau_{d}\right) e^{-\frac{x^{2}}{4 a^{2}\left(\tau_{d}\right)}}
$$

It evolves by spreading out according to the invariant Schrodinger's equation such that its dispersion increases which, at any instant $\tau$, between the slit and the screen, shall be noted $b(\tau)$, where $b^{2}(\tau)>a^{2}\left(\tau_{d}\right)$.

Suppose that at a proper time instant $\tau_{m}$, the useful part of the individual fabric reaches the screen. At this instant $\tau_{m}$, the transverse profile of the wavefunction is a normalised Gaussian $\varphi^{-}\left(\tau_{m}, x, y\right)$ presenting a dispersion $b\left(\tau_{m}\right)$, and has the following form:

$$
\varphi^{-}\left(\tau_{m}, x, y\right)=C\left(\tau_{m}\right) e^{-\frac{x^{2}}{4 b^{2}\left(\tau_{m}\right)}}
$$

The screen is a barrier that can be assimilated to a constant potential $V(x, y)=V_{0} \gg 1$ whereas, the zone of impact on the screen can be assimilated to an infinitesimal well. Then, at the proper instant $\tau_{m}$ of impact, the potential of the screen is equal 
to $V_{0}$ everywhere except within the zone of contact with the useful part of the wavefunction. This zone of contact can be represented by an infinitesimal Gaussian well of width $\Delta_{x} \approx 2 \varepsilon$ such that its associated contracting function is a localising Gaussian with an infinitesimally small and minimal dispersion $\varepsilon$ around a certain spatial point $\left(X_{0}, Z_{0}\right)$ within the zone of contact.

In particular, the transverse profile of the contracting function at the transverse point $X_{0}$ within the zone of contact can be expressed by a localising Gaussian, as follows:

$$
g_{X_{0}}(x)=\frac{1}{\left(2 \pi \varepsilon^{2}\right)^{1 / 4}} e^{-\frac{\left(x-X_{0}\right)^{2}}{4 \varepsilon^{2}}}
$$

Where the dispersion $\varepsilon$ of the localising Gaussian is much smaller than the dispersion $b\left(\tau_{m}\right)$ of the invariant wavefunction $\varphi^{-}\left(\tau_{m}, x, y\right)$.

The localising Gaussian instantaneously reduces the dispersion of the invariant wavefunction to $\varepsilon$. Indeed, the transverse profile of the antecedent invariant wavefunction $\varphi^{-}\left(\tau_{m}, x, y\right)$ having a dispersion $b\left(\tau_{m}\right)$ much larger than the zone of contact $2 \varepsilon$, is instantaneously transformed into a subsequent normalised Gaussian $\varphi^{+}\left(\tau_{m}, x, y\right)$ presenting a dispersion equal to the radius $\varepsilon$ of the zone of contact, as follows:

$$
\varphi^{+}\left(\tau_{m}, x, y\right)=D\left(\tau_{m}\right) e^{-\frac{\left(x-X_{0}\right)^{2}}{4 \varepsilon^{2}}}
$$

Thus, the impact of the useful part of the invariant wavefunction on the screen contracts this useful region to a localised condensed extension $2 \varepsilon$, making it behave as a corpuscle and, in particular, as a particle in an extremely narrow well.

The impact of the useful part of the individual fabric with the screen produces a complete transition of the density of the wavefunction from a stretched state to a localised state. This is similar to the localising function in the GRW theory, except that it is not a random spontaneous collapse but rather a response to the interaction between the wavefunction and the screen.

To estimate the localised condensed extension $2 \varepsilon$, it is known [33] in standard quantum mechanics that for a non-relativistic particle within a well of width $\Delta_{x} \approx 2 \varepsilon$, its momentum in the ground state $(n=1)$ is of the order:

$$
\hbar k=\hbar \pi / 2 \varepsilon
$$

Where

$$
k=\sqrt{\frac{2 m E}{\hbar^{2}}}
$$

Therefore, the radius of the well or the dispersion $\varepsilon$ of the contracting Gaussian should be of the order:

$$
\varepsilon \sim \frac{\pi \hbar}{\sqrt{8 m E}}=\frac{h}{\sqrt{2 m E}}
$$

We note that the uncertainty in momentum is $\Delta_{p} \approx \hbar \pi / \varepsilon$ and the uncertainty in position is $\Delta_{x} \approx 2 \varepsilon$ and thus, their product $\Delta_{x} \Delta_{p} \approx 2 \hbar \pi$ is consistent with the uncertainty principle.

By injecting the parameters relative to an electron having, for example, an energy $\mathrm{E}$ of $600 \mathrm{eV}$ in Equation (50), we obtain a dispersion $\varepsilon$ of the order of $10^{-11} \mathrm{~m}$.
It should be noted that if the barrier in the second period had two slits centred at $x=a$ and $x=-a$, forming a transverse double-square potential, then the induced contracting function $\gamma(x)$ would have concentrated the density of the wavefunction within the widths of both slits. The transverse contracting function $\gamma(x)$ can be approximated by a double-Gaussian centred at $x=a$ and $x=-a$, each of which presents a dispersion equal to the slit width $a$. The transverse profile of the wavefunction just downstream of the double-slit screen would thus be a superposition of two Gaussians centred at $x=a$ and $x=-a$. As the two Gaussians representing the distribution of the fabric's density spread out and overlap, an interference pattern would be created.

\section{MEASUREMENT}

In view of the above, measurement may not be the right term to use because the outcome of a measurement is not a pre-existing value. In particular, the measurement of the position of a physical system is a transformation like all others; the only special thing is that it transforms a pre-existing fabric having a stretched density into a fabric that has a highly localised density. This transformation is triggered by the introduction of a confining potential. Measurement can thus be formalised by multiplying the wavefunction $\varphi^{-}(\tau, r)$ with a localising Gaussian $g_{r_{0}}(\tau, r)$, as follows:

$$
\varphi^{+}(\tau, r)=g_{r_{0}}(\tau, r) \varphi^{-}(\tau, r)
$$

Consequently, the individual fabric exhibits a wave-like behaviour when most of its density is in a stretched state and a particle-like behaviour when most of its density is in a minimally contracted or localised state.

Moreover, the invariant Schrodinger's equation is always applicable, and discontinuity arises only because the process of interaction or measurement modifies the potential in the equation.

In general, the wavefunction $\varphi\left(\tau, r_{1}, r_{2}, \ldots r_{k}\right)$ of a set of $\mathrm{k}$ particles represents a global fabric composed of a combination (i.e. entanglement) or a juxtaposition (i.e. non-entanglement) of the individual fabrics corresponding to the different particles.

Similar to the GRW theorem [14] and as explained in detail for a two-particle system in [1], if the wavefunction is in the nonentangled (i.e. factorizable) state, the localisation of a particular dense region of an individual fabric does not affect the other dense regions. A localising Gaussian $g_{r_{0}}(\tau, r)$ acts only on a corresponding individual wavefunction $\varphi\left(\tau, r_{j}\right)$ without affecting the other individual wavefunctions. However, if the wavefunction is in the entangled (i.e. non-factorizable) state, the localisation of a particular dense region within the global fabric affects all other dense regions. A localising Gaussian $g_{r_{0}}\left(\tau, r_{j}\right)$ relative to any dense region acts holistically on the global fabric and thus on all its dense regions.

In a macroscopic object, each particle's individual fabric is subject to a confining potential corresponding to a resultant action created by all the other individual fabrics. Indeed, the interaction between the different particles confines the dense part of each fabric (represented by its wavefunction) within the 
confining potential; thus, a macroscopic object is always in a localised state.

\section{DISCUSSION}

Each individual fabric extends throughout the entire space; thus, all the individual fabrics in the universe may be represented by a single universal wavefunction $\varphi(\tau, \mathrm{X}) \equiv \varphi\left(\tau, x_{1}, x_{2}, \ldots x_{k}, \ldots\right)$. The universal wavefunction $\varphi(\tau, \mathrm{X})$ corresponds to a universal spacetime fabric over a set of points $X_{\tau} \equiv(\tau, X)$ in a fourdimensional spacetime $\mathrm{R} \times \mathrm{R}^{3}$ where each point $X_{\tau}$ represents a common position of all the corresponding individual positions. The combination and/or juxtaposition of all the individual fabrics defines a universal density distribution $\rho\left(X_{\tau}\right)$ over these points $(\tau, X)$. Indeed, spacetime is shaped by all the density distributions of all the individual fabrics composing the Universe.

Each individual fabric may be considered as a cosmological continuum, such that the global fabric represents the universe with all its constituents (matter, energy, dark matter, dark energy, and vacuum). The book edited by Valeri Dvoeglazov [34] gives particular attention to the concept of vacuum or ether, and hints at an obvious relation between the ether and dark matter or dark energy. Indeed, as the density distribution of free particles continues to stretch, it may be speculated that dark matter is composed of completely free individual fabrics (i.e. free particles) such that the density distribution of each individual fabric is so rarefied that it does not interact with its environment, while the global distribution of all the individual fabrics still has a gravitational effect.

We note that there is a different promising approach based on process algebra. Sulis $[35,36]$ proposed a descriptive theory by assuming a generated reality following Whitehead's process theory [37]. Whitehead [37] considered a process to be a sequence of events having a coherent temporal structure in which relations between events are more fundamental than the events themselves. The process algebra model posits a fundamental level of finite, discrete events upon which the usual entities of quantum mechanics supervene. It is argued that information and information flow provide an ontology of this fundamental level. Higher level constructs such as energy, momentum, mass, and spacetime are all emergent from this fundamental level. The model is compatible with both quantum mechanics and special relativity. It produces the results of quantum mechanics while still maintaining causally local realism.

However, in this study, we adhere to a more classical approach where the wavefunction is considered to represent the real physical features of a physical system.

\section{REFERENCES}

1. Travis Norsen in his book. Foundations of Quantum Mechanics. Berlin, Germany: Springer (2017).

2. Albert Einstein. Zur Elektrodynamik Bewegter Körper (On the Electrodynamics of Moving Bodies). Annalen der Physik (1905) 17:891. doi:10.1002/andp.19053221004

\section{CONCLUSION}

The quantum state of a physical system can be described by an invariant wavefunction $\varphi(\tau, x)$ corresponding to a single quantum event at a specific proper time $\tau$ which is invariant for all observers. This single quantum event short-circuits the spacetime intervals between different arguments. In particular, the invariant wavefunction represents an ontological object consisting of an individual fabric that has its proper individuality and a finite density amplitude that vanishes at infinity. More generally, the invariant wavefunction of a set of physical systems is defined in a high-dimensional configuration space. However, the density distributions of all these individual fabrics form a global fabric defined in four-dimensional physical spacetime. The global fabric is composed of a combination or juxtaposition of individual fabrics, each of which is also defined in the four-dimensional physical space time, and its identity is conserved.

Quantum measurement of the position of a physical system is a transformation caused by the introduction of a confining potential that triggers an instantaneous contraction of the dense region of the individual (or global) fabric. It is an inherent non-local transformation within the fabric itself, whereas the movement of the fabric relative to all others (i.e. with respect to the spacetime fabric) is local.

The author has declared that no competing interests exist.

All relevant data are within the paper.

This research did not receive any specific grant from funding agencies in the public, commercial, or not-for-profit sectors.

\section{DATA AVAILABILITY STATEMENT}

The original contributions presented in the study are included in the article/Supplementary Material, further inquiries can be directed to the corresponding authors.

\section{AUTHOR CONTRIBUTIONS}

I am the unique author (SY).

\section{ACKNOWLEDGMENTS}

I wish to thank Jérôme Lacaille, Jean Bricmont, Maurice Courbage and Eric Augard for many valuable, very helpful and fruitful discussions. I also wish to thank the reviewers who provided helpful commentary and additional references.

3. Maudlin T. Philosophy of Physics: Space and Time. Princeton, NJ: Princeton University Press (2012).

4. Einstein A, Podolsky B, Rosen N. Can Quantum-Mechanical Description of Physical Reality Be Considered Complete? Phys Rev (1935) 47(10):777-80. doi:10.1103/physrev.47.777

5. Bell JS. On the Einstein Podolsky Rosen Paradox. Physics (1964) 1(3):195-200. doi:10.1103/physicsphysiquefizika.1.195

6. Bell JS. On the Problem of Hidden Variables in Quantum Mechanics. Rev Mod Phys (1966) 38:447-52. doi:10.1103/RevModPhys.38.447 
7. Aspect A, Grangier P, Roger G. Experimental Realization of EinsteinPodolsky-Rosen-BohmGedankenexperiment: A New Violation of Bell's Inequalities. Phys Rev Lett (1982) 49(2):91-4. doi:10.1103/ physrevlett.49.91

8. Felline L. On Explaining Quantum Correlations: Causal vs. Non-causal. Entropy (2021) 23:589. doi:10.3390/e23050589

9. Thomsen K. Timelessness Strictly Inside the Quantum Realm. Entropy (2021) 23:772. doi:10.3390/e23060772

10. Drummond B. Understanding Quantum Mechanics: A Review and Synthesis in Precise Language. Open Phys (2019) 17:390-437. doi:10.1515/phys-20190045

11. Everett H. The Theory of the Universal Wave Function. Thesis. Princeton, NJ: Princeton University (1956).

12. de Broglie L. Remarques sur la théorie de l'onde-pilote. C.R Acad Sci Paris (1951) 233:641-4.

13. Bohm D. A Suggested Interpretation of the Quantum Theory in Terms of "Hidden" Variables. I. Phys Rev (1952) 85:166-79. doi:10.1103/ physrev.85.166

14. Ghirardi GC, Rimini A, Weber T. Unified Dynamics for Microscopic and Macroscopic Systems. Phys Rev D (1986) 34:470-91. doi:10.1103/ physrevd.34.470

15. Wallace D. Emergent Multiverse. Oxford, UK: Oxford (2012).

16. Kent A. One World vs. Many: The Inadequacy of Everettian Accounts of Evolution, Probability, and Scientific Confirmation. arXiv: quant-ph/ $0905.0624 v 3$ (2013).

17. Greaves H. Understanding Deutsch's Probability in a Deterministic Multiverse. Stud Hist Philos Sci B: Stud Hist Philos Mod Phys (2004) 35: 423-56. doi:10.1016/j.shpsb.2004.04.006

18. Bricmont J. Making Sense of Quantum Mechanics. Berlin, Germany: Springer (2016).

19. Durt T, Fargue D, Matzkin A, Robert J. Symposium Louis de Broglie. Ann de la Fondation Louis de Broglie (2021) 46(1).

20. Colin S, Durt T, Willox R, de L. Broglie's Double Solution Program: 90 Years Later. Ann de la Fondation Louis de Broglie (2017) 42:19.

21. Mielnik B. The Paradox of Two Bottles in Quantum Mechanics. Found Phys (1990) 20:745-55. doi:10.1007/bf01889459

22. Helwig KE, Kraus K. Formal Description of Measurements in Local Quantum Field Theory. Phs Rev (1970) D1:566. doi:10.1103/PhysRevD.1.566

23. Aharonov Y, Albert DZ. States and Observables in Relativistic Quantum Field Theories. Phys Rev D (1980) 21:3316-24. doi:10.1103/physrevd.21.3316

24. Aharonov Y, Albert DZ. Can We Make Sense Out of the Measurement Process in Relativistic Quantum Mechanics? Phys Rev D (1981) 24:359-70. doi:10.1103/physrevd.24.359
25. Finkelstein J. Covariant Collapse of the State Vector and Realism. Found Phys Lett (1992) 5(4):383. doi:10.1007/bf00690595

26. Minkowski H. Raum und Zeit. Jahresberichte der deutschen MathematikerVereinigung (1909) 18:75.

27. Gourgoulhon E. Special Relativity in General Frames. Berlin, Germany: Springer (2013).

28. Wigner E. Group Theory and its Application to the Quantum Mechanics of Atomic Spectra. New York, NY: Academic Press (1959).

29. Loprez C. Seeking for a Fundamental Quantum Arrow of Time. Front Phys (2018) 6:104. doi:10.3389/fphy.2018.00104

30. Albert DZ. Time and Chance. Cambridge, MA: Harvard University Press (2000).

31. Callender C. Is Time 'Handed' in a Quantum World? Oxford, UK: Poc Aristotelian Soc. (2000).

32. Maudlin T. Philosophy of Physics: Quantum Theory. Princeton, NJ: Princeton University Press (2019).

33. Binney J, Skinner D. The Physics of Quantum Mechanics. Oxford, UK: Oxford (2014).

34. Dvoeglazov V. Einstein and Poincaré: The Physical Vacuum. Montréal: Apeiron (2006).

35. Sulis W. Locality Is Dead! Long Live Locality!. Front Phys (2020) 8:360. doi: $10.3389 /$ fphy. 2020.00360

36. Sulis W. An Information Ontology for the Process Algebra Model of Nonrelativistic Quantum Mechanics. Entropy (Basel) (2020) 22(2):136 doi:10.3390/e22020136

37. Whitehead AN. Process and Reality. New York, NY: Free Press (1978).

Conflict of Interest: The author declares that the research was conducted in the absence of any commercial or financial relationships that could be construed as a potential conflict of interest.

Publisher's Note: All claims expressed in this article are solely those of the authors and do not necessarily represent those of their affiliated organizations, or those of the publisher, the editors and the reviewers. Any product that may be evaluated in this article, or claim that may be made by its manufacturer, is not guaranteed or endorsed by the publisher.

Copyright (c) 2021 Yasmineh. This is an open-access article distributed under the terms of the Creative Commons Attribution License (CC BY). The use, distribution or reproduction in other forums is permitted, provided the original author(s) and the copyright owner(s) are credited and that the original publication in this journal is cited, in accordance with accepted academic practice. No use, distribution or reproduction is permitted which does not comply with these terms. 